\title{
A RARE CASE OF HUNTER SYNDROME - CASE REPORT
}

\author{
Lorena Elena Melit, Oana Marginean, Carmen Duicu, Cristina Campean, \\ Maria Oana Marginean \\ Paediatric Clinic, University of Medicine and Pharmacy Tg. Mures, Romania
}

\begin{abstract}
Mucopolysaccharidoses (MPSs) are a group of rare genetic disorders within the larger family of lysosomal diseases. MPSs disorders are caused by a deficiency in the activity of a specific lysosomal enzyme required for the degradation of glycosaminoglycans (GAGs). MPS type II, also called Hunter syndrome consists in a deficiency of an enzyme, iduronate-2-sulphatase.

We present a rare case of Hunter syndrome with atypical presentation. It is a case about a boy of 2.7 year old who presented to the Paediatric Clinic with symptoms of a respiratory tract infection and a history of frequent ear infections and respiratory tract infections. His clinical examination showed the following abnormalities: high stature, moderately overweight, facial dismorphism (coarse facial features, prominent forehead, a depressed nasal bridge), small stubby fingers with flexion of distal interphalangeal joints, joint stiffness, protruding abdomen with umbilical hernia, hepatomegaly and splenomegaly, and also mild mental retardation.

The clinical aspect was suggestive for MPS type I, II or VII. Dosage of enzymes with role in mucopolysaccharide lysosomal metabolism revealed low levels of iduronate-2-sulphatase, changes that bent for MPS type II. Enzymatic diagnosis was confirmed by molecular DNA analysis that showed a hemizygote mutation of iduronate2-sulphatase gene in intron 3 (c.419-2A > G). Enzyme replacement therapy with recombinant human iduronate2-sulfatase (Elaprase ${ }^{\circledR}$ ) was started.

The evolution of the case after almost two years of treatment with Elaprase is favorably, without loss of neurological acquisitions.
\end{abstract}

Keywords: Hunter syndrome, child

\section{INTRODUCTION}

Mucopolysaccharidosis type II or Hunter syndrome is a lysosomal storage, which results from the deficiency of the enzyme called iduronate2-sulphatase (IDS). This syndrome is genetically inherited, having X-linked recessive inheritance, and it consists in a homozygote mutation of the IDS gene, located on the chromosome Xq28, thus leading to the affliction of the synthesized protein. This enzyme being responsible for the degradation of heparan and dermatan sulphate, its absence will lead in the case of afflicted patients to the accumulation of these substances in different tissues, determining in this way also the clinical picture of this disease $(1,2)$. The clinical picture of this syndrome includes cardio-vascular signs and symptoms, as the involvement as myocardium and the valve thickening; neuro-psychiatric disorders: behavioral disorders, cervical myelopathy, hydrocephaly, mental retardation, acquisitions regression, seizures and deglutition difficulties; digestive signs and symptoms: diarrhea, hepatosplenomegaly; year involvement: recurrent medium otitis, deafness; eye involvement, meaning retinal dystrophy; muscle and bones disorders: atlanto-axial instability, carpal tunnel syndrome, facial dysmorphism, degenerative hip dysplasia, disostosis multiplex, hernias, joint contractions, kyphosis and macrocephaly; and also respiratory symptoms: obstructive sleep apnea, restrictive pneumopathy, obstruction of the upper airways (3-7). Though the diagnosis of this disease is mainly a clinical one, its confirmation supposes also many paraclinical investigations (blood tests, X-rays, CT, MRI, DEXA, EKG, ecocardiography, neurological consult, ear-nose-throat 
specialist consult, ophthalmologist consult), and not ultimately, the molecular analysis of the IDS gene, with major importance. The specific treatement of mucopolysaccharidosis type II is just a substitutive one, without being curative, and it consists in the administration of the enzyme iduronate2-sulphatase.

Throughout the presentation of this case, we want to underline the atypical clinical picture of this patient, with mild mental retardation without corneal impairment, respectively the diagnosis and treatment problems raised by this case.

\section{CASE PRESENTATION}

A 2 years and 7 months old boy presented at the Pediatrics Clinic from Targu Mures, with a history of multiple infections of the upper airways and frequent otitis.

The pathological elements of the clinical examination were: high stature (SDS+3.46), moderate overweight $(+25.97 \%)$, short neck, facial dysmorphism, prominent forehead, depressed nasal bridge, small, stubby fingers, with the flexion of distal interphalangeal joints, joint stiffness, distended abdomen, with umbilical hernia, hepatosplenomegaly, mild mental retardation.
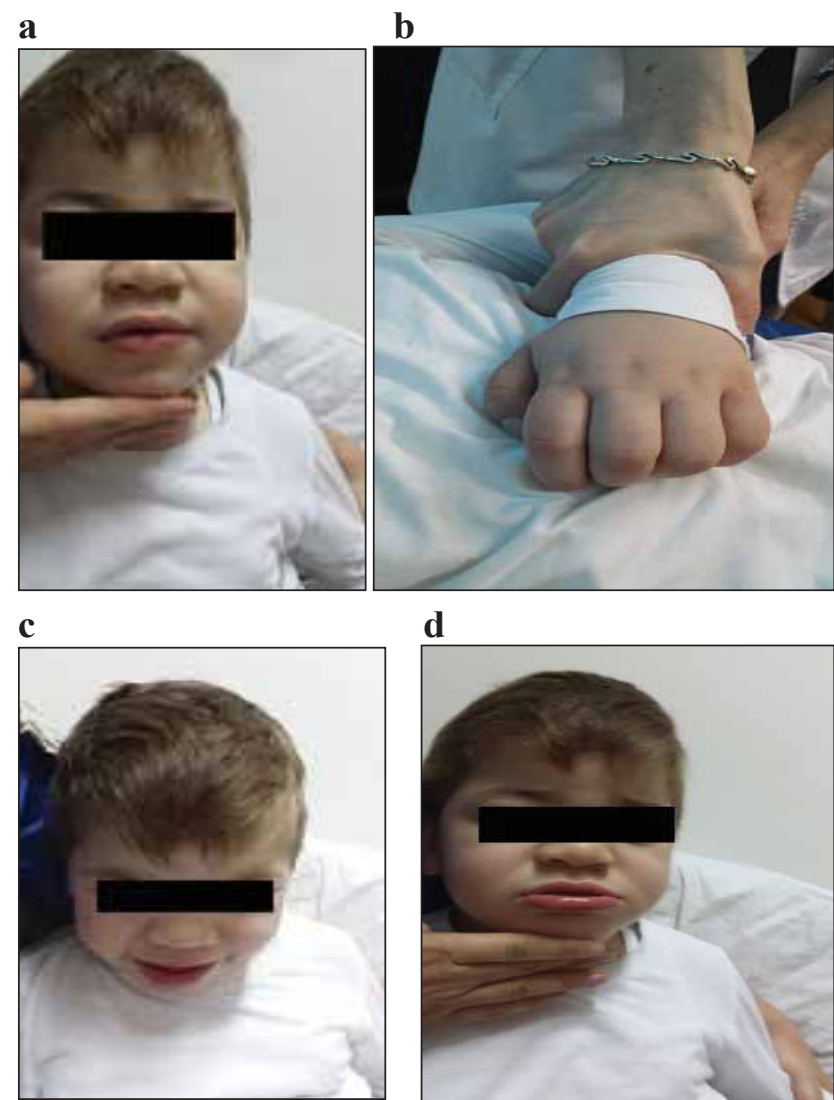

d

FIGURE 1. a, b, c, d: Physical features characteristic for Hunter syndrome in the presented case
The clinical picture of this patient was suggestive for mucopolysaccharidosis type I, II or VII, reason for which there were performed paraclinical investigation for elucidating the diagnosis. Thus the enzyme dosage consisted in: Cl. Mainz plasma iduronate sulpahatase with a value of $6.7986 \mathrm{nM} /$ $\mathrm{ml} / 24 \mathrm{~h}$ (normal values: $300-800 \mathrm{nM} / \mathrm{ml} / 24 \mathrm{~h}$ ) and Cl. Rostock plasma iduronate sulphatase with a value of $1.3 \mu \mathrm{mol} / \mathrm{l} / \mathrm{h}$ (normal values $\geq 2$ ). The molecular analysis of IDS gene revealed a homozygote mutation of intron 3 (c.419-2A>G). Thus the diagnosis of type II mucopolysaccharidosis was confirmed. The only pathological element pointed out by the biochemical and hematological blood tests was the value og IgA, which was $0 \mathrm{mg} / \mathrm{dl}$. There were also performed multiple X-rays, meaning: fist and hand X-ray, which showed proper bone age for 2 years and faced methaphises; thoracal X-ray was without pleuro-pulmonar modifications, without pathological modifications of the heart, with slimmer costal arcs at the costo-vertebral joints; spinal column radiography underlined a tendency for lordosis, lumbar vertebral bodies with anterior aspect of beak; pelvic X-ray with the following pathological aspects: 'chalice' shaped pelvis, with iliac bones bigger than the ischio-pubic ones, horizontal cotiloid brown, deformed, bent for bilateral coxo-femoral contortion, more expressed on the left, bilateral coxa valga; and finally the $\mathrm{X}$-ray of the forearm and hand showed forearm bones with shorter diaphises, carpal bones with angled diaphisis, distal phalanges in flexion. Thus all this radiological elements were suggestive type II mucopolysaccharidosis. Ultrasonographic examinations, meaning echocardiography and abdominal ultrasound revealed first degree aortic insufficiency, first degree mitral insufficiency, mild ventricular hypertrophy and mild hepato-splenomegaly. The consult at the ophthalmologist with bottom eye examination pointed out a contracted papilla, with a small central excoriation of the right eye, normal caliber vessels, free macula, without reflex, thin retina with the visibility of the choroidian array. There was also performed a consult at the ear-nose-throat specialist, but without any pathological elements. The neurologist and psychiatrist specialist established the diagnosis of mild mental retardation.

Our diagnosis of type II mucopolysaccharidosis was sustained by the clinical picture, the paraclinical investigations and confirmed by the genetic test.

Once we established the diagnosis, the substitutive enzyme treatment with Elaprase was initiated (the purified form of iduronate-2-sulphatase) at a 


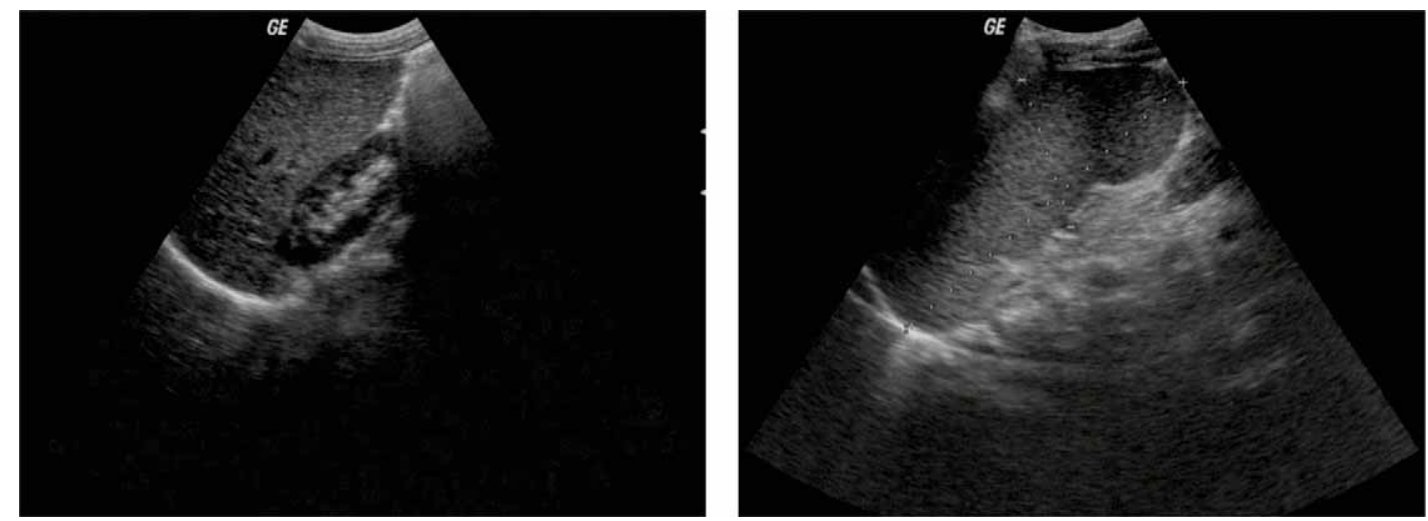

FIGURE 2. a, b. Hepatomegaly and splenomegaly highlighted ultrasound

dose of $0.5 \mathrm{mg} / \mathrm{kg} / \mathrm{dose} /$ week, which is administered weekly in the Pediatrics Clinic I of Emergency Clinical County Hospital of Târgu-Mures. The evolution of this case after 2 years from the diagnosis is a stationary one, but unfortunately the prognosis of this case is unfavorable, because this disease is without curative treatment, and the substitutive treatment was initiated late, after the settlement of the neuro-psychiatric retardation and after the appearance of the behavioral disorders.

\section{DISCUSSIONS}

Mucopolysaccharidoses represent a group of rare genetic disorders, that belong to the great family of the lysosomal storage disorders. There described 11 types of mucopolysaccharidoses, each of them consisting in a specific enzyme deficiency for the degradation of glicozaminoglicans, named also mucopolysaccharidosis, leading in this way to the accumulation of those in the tissues, and as a result to progressive and permanent cellular death, reducing the length of life $(8,9)$. Type II mucopolysaccharidosis is named also Hunter syndrome after the name of the Canadian physician, Charles Hunter, who in the year 1917, described a rare disease in two brothers $(3,4)$. This syndrome is one the most frequent mucopolysaccharidosis, with a prevalence of one at 170.000 male new-borns, the presented patient in our case being also a male. In Romania there are 8 cases diagnosed with Hunter syndrome, the one presented by us being the seventh one. There are two forms of type II mucopolysaccharidosis, a mild one and a severe one, classified depending on the impairment of the central nervous system (7). Thus, in the severe form, the death appears usually in the second decade of age, and the main causes are represented by the upper airway obstruction or by the cardiac insufficiency (6), this is why the early acknowledgement of the airways impairment is very important in patients with Hunter syndrome, and it can guide the treatment (10). Regarding the cardiac affliction, the absence of abnormal precordial sounds does not rule out the cardiopathy, thus the echocardiography ant the electrocardiogram are the key methods in diagnosing this disorder (11). In mild forms, the patients do not usually present the impairment of the central nervous system, or if it exist, this is minor, otherwise the organic manifestations became very severe until the adulthood, leading to psychological problems or difficult adaptation in the society (12). According to the clinical picture, our patient was classified as having the mild form of this syndrome, but with a not very good prognosis because the substitutive treatment was initiated late, after the appearance of neurological and psychiatric retardation.

The enzyme substitutive therapy with human recombinant iduronate-2-sulphatase, would lead to reducing the volume of liver and spleen (13), and also to the improvement of the respiratory and motor functions $(14,15)$. Though the enzyme substitutive treatment is a specific one, the psysician must also pay attentios to the supportive treatment in this patients case because the complications of this pathology are plenty and diverse.

\section{CONCLUSIONS}

Mucopolysaccharidoses are multisistemic disorders, for whom diagnosis and management being needed a multidisciplinary team, meaning a pediatrician or internal medicine specialist, a radiologist, a genetician, an ear-nose-throat specialist, an ophthalmologist, a neurologist, and in particular extreme cases even a surgeon and an intensive care specialist. The early diagnosis, and respectively, the treatment initiated as soon as possible, are recommended for preventing the evolution of this dis- 
order and the appearance of the complications. Even though Hunter syndrome, as al mucopolysaccharidoses, does not benefit nowadays from a cura- tive treatment, the improvement of life quality of this patients, must be accounted between the main objectived of this rare genetic disorder.

\section{REFERENCES}

1. Peining L., Bellows A.B., Thompson J.N. Molecular basis of iduronate-2-sulfatase gene mutations in patients with mucopolysaccharidosis type II (Hunter syndrome). J Med Genet 1999; 36:21-27.

2. Burruss D.M., Wood T.C., Espinoza L. et al. Severe Hunter Syndrome (Mucopolysaccharidosis II) Phenotype Secondary to Large Deletion in the $X$ Chromosome Encompassing IDS, FMR1, and AFF2 (FMR2). J Child Neurol 2012; 27:786-790.

3. Wraith J.E., Scarpa M., Beck M. et al. Mucopolysaccharidosis Type II (Hunter Syndrome): A clinical review and recommendations for treatment in the era of enzyme replacement therapy. Eur J Pediatr 2008; 167:267-77.

4. Martin R., Beck M., Eng C., Giugliani R. et al. Recognition and diagnosis of mucopolysaccharidosis II (Hynter syndrome). Pediatrics 2008; 121:377-86.

5. Kliegman R.M., Behrman R.E., Jenson H.B., Stanton F.B. Nelson Textbook of Pediatrics, 18th ed, vol 1. Philadelphia: Saunders, 2007.

6. Tuschl K., Gal A., Paschke E. et al. Mucopolysaccharidosis Type II in females: Case report and review of literature. Pediatr Neurol 2005; $32: 270-2$

7. Shah G.S., Mahal T., Sharma S. Atypycal clinical presentation of mucopolysaccharidosis type II (Hunter syndrome): A case report. J Med Case Reports 2010;4:154.

8. Gajula P., Ramalingam K., Bhadrashetty D. A rare case of mucopolysaccharidosis: Hunter sydrome. J Nat Sci Biol Med 2012; 3:97-100

9. Burton B.K., Giugliani R. Diagnosing Hunter syndrome in pediatric practice: practical considerations and common pitfalls. Eur J Pediatr 2012; 171:631-639.

10. Yeung A.H., Cowan M.J., Horn B., Rosbe K.W. Airway Management in Children With Mucopolysaccharidoses. Arch Otolaryngol Head Neck Surg 2009; 135:73-79.

11. Braunlin E.A., Harmatz P.R., Scarpa M. et al. Cardiac disease in patients woth mucopolysaccharidosis: presentation, diagnosis and management. J Inherit Metab Dis 2011; 34:1183-97.

12. Kuratsubo I., Suzuki Y., Orii K.O. et al. Psychological status of patients with mucopolysaccharidosis type II and their parents. Pediatrics International 2009; 51:41-47.

13. Muenzer J., Beck M., Gugliani R. et al. Idursulfase treatment of Hunter syndrome in children younger than 6 years: Results from the Hunter Outcome Survey. Genet Med 2011; 13:102-109.

14. Muenzer J., Wraith J.E., Beck M. et al. A phase II/III clinical study of enzyme replacement therapy with idursulfase in mucopolysaccharidosis II (Hunter syndrome). Genet Med 2006; 8:465-73.

15. Muenzer J., Gucsavas-Calikoglu M., McCandless S. et al. A phase I/II clinical trial of enzyme replacement therapy woth idursulfase in mucopolysaccharidosis II (Hunter syndrome). Mol Genet Metab 2007; 90:329-37. 